\title{
Rare earth elements and carbon isotope geochemistry of the Doushantuo Formation in South China: Implication for middle Ediacaran shallow marine redox conditions
}

\author{
ZHOU ChuanMing $^{1 *}$, JIANG ShaoYong $^{2}$, XIAO ShuHai $^{1,3}$, CHEN Zhe $^{1}$ \& YUAN XunLai ${ }^{1}$ \\ ${ }^{1}$ State Key Laboratory of Palaeobiology and Stratigraphy, Nanjing Institute of Geology and Palaeontology, Chinese Academy of Sciences, \\ Nanjing 210008, China; \\ ${ }^{2}$ State Key Laboratory for Mineral Deposits Research, Department of Earth Sciences, Nanjing University, Nanjing 210093, China; \\ ${ }^{3}$ Department of Geosciences, Virginia Polytechnic Institute and State University, Blacksburg, VA 24061, USA
}

Received January 11, 2012; accepted February 20, 2012; published online March 23, 2012

\begin{abstract}
The middle Ediacaran Shuram excursion, the largest negative $\delta^{13} \mathrm{C}_{\text {carb }}$ excursion in Earth history, has been interpreted as indirect evidence for episodic oxidation and remineralization of deep ocean DOC (dissolved organic carbon). It has been hypothesized that such oxidation event may have occurred when anoxic DOC-laden deep water was brought to shallow shelves during oceanic upwelling, which is expected to cause localized anoxia in shallow environments. To test this prediction, we systematically analyzed rare earth elements (REE) and $\delta^{13} \mathrm{C}_{\text {carb }}$ of the upper Doushantuo Formation carbonates in the Yangtze Gorges area of South China, which were deposited in an inner shelf environment and record a large negative $\delta^{13} \mathrm{C}_{\text {carb }}$ excursion correlated to the Shuram event. The REE data show a significant positive shift in $\mathrm{Ce} / \mathrm{Ce} e^{*}$ values, synchronous with a pronounced negative $\delta^{13} \mathrm{C}_{\text {carb }}$ shift. This positive $\mathrm{Ce} / \mathrm{Ce}$ * shift is interpreted to represent an oceanic anoxia event in shallow shelf environments, which may have been caused by the upwelling or impingement of oxygen-depleted and ${ }^{12} \mathrm{C}$-enriched deep water onto shelves. This anoxia event coincides with a sharp decline in the abundance and diversity of Ediacaran acanthomorphic acritarchs, raising the possibility that these two geobiological events may be causally related.
\end{abstract}

REE, marine anoxia, Doushantuo Formation, Yangtze Gorges, South China

Citation: Zhou C M, Jiang S Y, Xiao S H, et al. Rare earth elements and carbon isotope geochemistry of the Doushantuo Formation in South China: Implication for middle Ediacaran shallow marine redox conditions. Chin Sci Bull, 2012, 57: 1998-2006, doi: 10.1007/s11434-012-5082-6

Carbon and sulfur isotope data indicate that deep oceans were episodically oxygenated and deep ocean DOC progressively remineralized during the Ediacaran Period $[1,2]$. These remineralization events likely involved upwelling of anoxic DOC-laden water to shallow shelves, impacting on the shallow-water redox conditions and biological evolution. Indeed, the Ediacaran fossil record shows a significant biotic turnover in shallow shelf environments during the middle Ediacaran Period, with the early Ediacaran biota characterized by globally distributed acanthomorphic acritarchs [3], and the late Ediacaran ones by classical Ediacara megafossils [4]. Previous researchers have argued that this

*Corresponding author (email: cmzhou@ nigpas.ac.cn) turnover may have been driven by a middle Ediacaran glaciation event [5], predation pressure from macrophagous metazoans [3], or the oxygenation of continental shelves that released the ecological impetus for the development of acanthomorphic resting stages [6]. Integrated paleobiological and geochemical data from the Doushantuo Formation in the Yangtze Gorges area suggest that this turnover is coincident with a pronounced negative $\delta^{13} \mathrm{C}_{\text {carb }}$ excursion that is correlated with the Shuram excursion [7], and thus may be related to shallow water anoxia resulting from upwelling of deep marine waters.

The Doushantuo Formation (635-551 Ma in age, including four lithostratigraphic members I-IV in ascending order; Figure 1) in the Yangtze Gorges area was considered to 
have been deposited mostly in a shelf lagoon separated from the open ocean by a shelf margin barrier [8], but others $[9,10]$ suggested that the middle and upper Doushantuo Formation in the Yangtze Gorges area, including Member III - the focus of this paper, might have been deposited in an open shelf. Previous studies indicate that in the Yangtze Gorges area Ediacaran acanthomorphs appear shortly after the termination of the Nantuo glaciation and disappear in Member III of the Doushantuo Formation, just below the pronounced negative $\delta^{13} \mathrm{C}_{\text {carb }}$ excursion EN3 (Figure 1) that is correlated with the Shuram excursion and represents the largest negative $\delta^{13} \mathrm{C}_{\text {carb }}$ excursion in Earth history [2,3,7,11]. After EN3, Ediacara-like megafossils appear in the Dengying Formation [12-15]. Thus, Member III represents a critical interval to understand the middle Ediacaran evolutionary turnover and its environmental context.

Cerium (Ce) is the only rare earth element (REE) that may exist in either trivalent or tetravalent forms in natural environments. Ce (III) can be oxidized to insoluble Ce (IV) in modern oxygenated seawater, which results in negative $\mathrm{Ce}$ anomaly because $\mathrm{Ce}$ (IV) is removed through absorption onto particles of $\mathrm{Fe} / \mathrm{Mn}$-oxyhydroxides. In contrast, $\mathrm{Ce}$ (IV) is reduced to soluble Ce (III) in suboxic and anoxic conditions, leading to normal $\mathrm{Ce}$ concentration or apparent $\mathrm{Ce}$ enrichment compared to its neighbors [16]. The original seawater REE patterns may be retained in ancient non-skeletal carbonates [17](but see German and Elderfield [16] for different view), thus Ce anomaly of carbonates can be used to trace seawater redox changes through geological time. In this paper, we investigate $\mathrm{Ce}$ anomalies of carbonates from the Member III of the Doushantuo Formation. Together with the pronounced $\delta^{13} \mathrm{C}_{\text {carb }}$ excursion, we apply

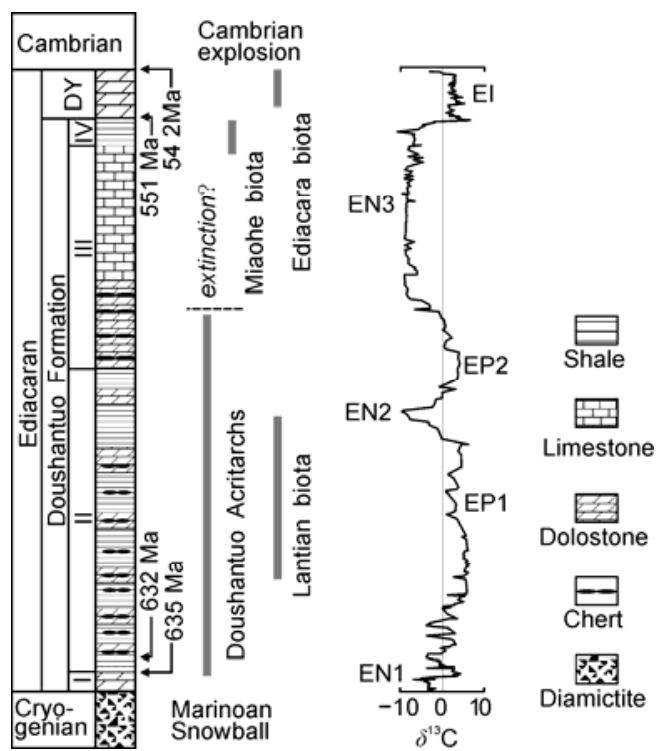

Figure 1 Simplified lithostratigraphic column, fossil distribution, and $\delta^{13} \mathrm{C}_{\text {carb }}$ chemostratigraphy of the Ediacaran System in the Yangtze Gorges area, South China. $\delta^{13} \mathrm{C}_{\text {carb }}$ curve modified from Jiang et al. [18] and McFadden et al. [2]. DY, Dengying Formation.
Ce anomaly to evaluate the redox condition on the shallow shelf of the Yangtze platform, South China.

\section{Lithostratigraphy and fossil occurrence}

We studied Jiulongwan and Tianjiayuanzi sections of the Ediacaran Doushantuo Formation from the Yangtze Gorges area in this paper. Overlying the Cryogenian Nantuo Formation diamictite, the Ediacaran Doushantuo Formation in the Yangtze Gorges area consists of four lithological members. Member I is mainly composed of dolostone (i.e., cap dolostone). The overlying Member II is characterized by black shales interbedded with dolostones. Member III at the well-studied Jiulongwan section consists of $\sim 25 \mathrm{~m}$ thick medium- to thick-bedded dolostones with thin sheet-like chert horizons and irregular chert nodules (Figure S1h), followed by $\sim 7 \mathrm{~m}$ thick thin-bedded dolostones and then $\sim 24 \mathrm{~m}$ thick limestone with thin dolostone interbeds (Figure 2; Figure $\mathrm{S} 1 \mathrm{~b}, \mathrm{f}, \mathrm{g})$. The lithologic transition in Member III may suggest a slightly deepening-upward sequence, although the occurrence of small scours and intraclastic/oncolitic beds (Figure S1a, c, e) throughout the entire member suggests deposition above fair-weather wavebase. At the Tianjiayuanzi section, Member III is $\sim 48 \mathrm{~m}$ thick and mainly consists of medium- to thick-bedded dolostones with thin chert layers (Figure 2; Figure S2g,h). Here, dolomitic ooids, phosphatic oncoids, silicified oncolites, and cross-bedding structures (Figure S2b-f) in the upper part of Member III indicate deposition in a shallow subtidal environment. The uppermost Member III at Tianjiayuanzi is composed of $\sim 3 \mathrm{~m}$ of thin-bedded dolostone, which may also suggest a deepening-upward sequence as the case at Jiulongwan, although the limestone unit seen at Jiulongwan is absent at Tianjiayuanzi. Member IV of the Doushantuo Formation in both the Jiulongwan and Tianjiayuanzi sections is mainly composed of black shales.

At Tianjiayuanzi, eight genera and eighteen species of acanthomorphic acritarchs have been recovered from early diagenetic cherts in Member II and III [3,11]. At Jiulongwan, acanthomorphs have also been found in both members [19]. It appears that acanthomorphs from Member II and III represent two distinct assemblages [11,19], but most acanthomorphs disappear as $\delta^{13} \mathrm{C}_{\text {carb }}$ values fall rapidly, i.e. in EN3 interval.

\section{Methods and results}

We systematically collected carbonate and chert samples from Member III at the Jiulongwan and Tianjiayuanzi sections for rare earth elements analysis, $\delta^{13} \mathrm{C}_{\text {carb }}$ chemostratigraphy, and acanthomorph biostratigraphic analysis. For each carbonate rock, a carefully selected fresh chip was ground in an agate mortar, and the powder was then used for carbon 
isotope and REE measurements. For carbon isotope measurements, $\mathrm{CO}_{2}$ was extracted using a standard offline technique, with samples reacting with $100 \% \mathrm{H}_{3} \mathrm{PO}_{4}$ at $50^{\circ} \mathrm{C}$ for $12 \mathrm{~h}$. Carbon and oxygen isotope ratios were determined on a Finnigan Delta Plus XP mass spectrometer. Analytical precision is better than $0.1 \%$ or for $\delta^{13} \mathrm{C}$ and $0.2 \%$ for $\delta^{18} \mathrm{O}$. Isotopic data are reported in per mil (\%o) vs. VPDB. For rare earth element analyses, $\sim 50 \mathrm{mg}$ powder of each sample was allowed to react with $0.5 \mathrm{~N}$ acetic acid at room temperature for $12 \mathrm{~h}$. A subset of samples was also treated with $1.0 \mathrm{~N}$ acetic acid to test the impact of contamination from terrigenous detritus. The rare earth element concentrations of the supernatant solutions were then determined on a Finnigan HR-ICP-MS. Analytical precision is better than $10 \%$. The remaining sample solutions after REE analyses were also measured for their $\mathrm{Mg}$ and $\mathrm{Ca}$ concentrations and $\mathrm{Mg} / \mathrm{Ca}$ ratios by ICP-OES method. In addition, an aliqout of supernatant solutions of selected samples was dried and re-dissolved using double-distilled super-clean $\mathrm{HNO}_{3}$ in Teflon vials overnight, and then $\mathrm{Sr}$ fractions were separated and purified using conventional cation exchange column. $\mathrm{Sr}$ was then loaded on single Ta filament sandwiched with a $\mathrm{TaF}_{5}$ solution. Sr isotope ratios were measured using a Finnigan Triton TI mass spectrometer. During the analysis period, measurement of the NIST-987 Sr standard yields results of ${ }^{87} \mathrm{Sr} /{ }^{86} \mathrm{Sr}=0.710262 \pm 0.000005(2 \sigma) . \mathrm{C}-\mathrm{O}$ and $\mathrm{Sr}$ isotopes, $\mathrm{Mg} / \mathrm{Ca}$ ratios and rare earth element analyses were all carried out at the State Key Laboratory for Mineral Deposits Research in Nanjing University.

The $\delta^{13} \mathrm{C}_{\text {carb }}$ data from the present study are similar to previous reports from the same stratigraphic interval in the Yangtze Gorges area [2,18] and elsewhere in South China [20], showing a major negative shift from $4.6 \%$ to $-9.3 \%$ o and from $4.7 \%$ to $-11.9 \%$ in the dolostone unit of Member III at Jiulongwan and Tianjiayuanzi, respectively (Figure 2). New $\delta^{13} \mathrm{C}_{\text {carb }}$ chemostratigraphic data show that the entire Member III at Tianjiayuanzi may be equivalent to only the lower part of Member III at Jiulongwan, which is consistent with the missing limestone unit at the former section.

The NASC-normalized REE distribution patterns for most carbonate samples are relatively flat, with slight LREE enrichment (average $\mathrm{Nd}_{\mathrm{SN}} / \mathrm{Yb}_{\mathrm{SN}}=1.31, \mathrm{SD}=0.57$ ) (Table 1; Table S1 and Figure S3). At Jiulongwan, Ce/Ce* values [calculated as $\mathrm{Ce}_{\mathrm{SN}} /\left(0.5 \mathrm{La}_{\mathrm{SN}}+0.5 \mathrm{Pr}_{\mathrm{SN}}\right)$; $\mathrm{SN}$ refers to NASC normalization] show a positive shift from 0.29 to 0.90 , synchronous with the negative $\delta^{13} \mathrm{C}_{\text {carb }}$ shift (Figure 2). At Tianjiayuanzi, as $\delta^{13} \mathrm{C}_{\text {carb }}$ shift from $4.7 \%$ o to $-4.7 \%$, $\mathrm{Ce} / \mathrm{Ce}^{*}$ values fluctuate between 0.02 and 0.67 . $\mathrm{Ce} / \mathrm{Ce}^{*}$ values reach $\sim 0.82$ in the uppermost part of Member III at Tianjiayuanzi, corresponding to $\delta^{13} \mathrm{C}_{\text {carb }}$ values between $-7.8 \%$ o and $-11.9 \%$ (Figure 2).

\section{Discussion}

\subsection{Validity of REE data}

Because REE concentrations are much higher in detritus than in seawater, terrigenous sediments could elevate REE contents and mask seawater signatures preserved in marine carbonates [17]. Thin section observation indicates that small amounts of phosphatic grains and terrigenous silts do occur in the analyzed carbonate samples (Figures S1a, c; $\mathrm{S} 2 \mathrm{a})$. However, the effect of terrigenous detritus was minimized because the samples were treated with a weak acid ( $0.5 \mathrm{~N}$ acetic acid), and this is consistent with the lack of correlations between $\mathrm{Zr}$ concentrations and Y/Ho ratios,

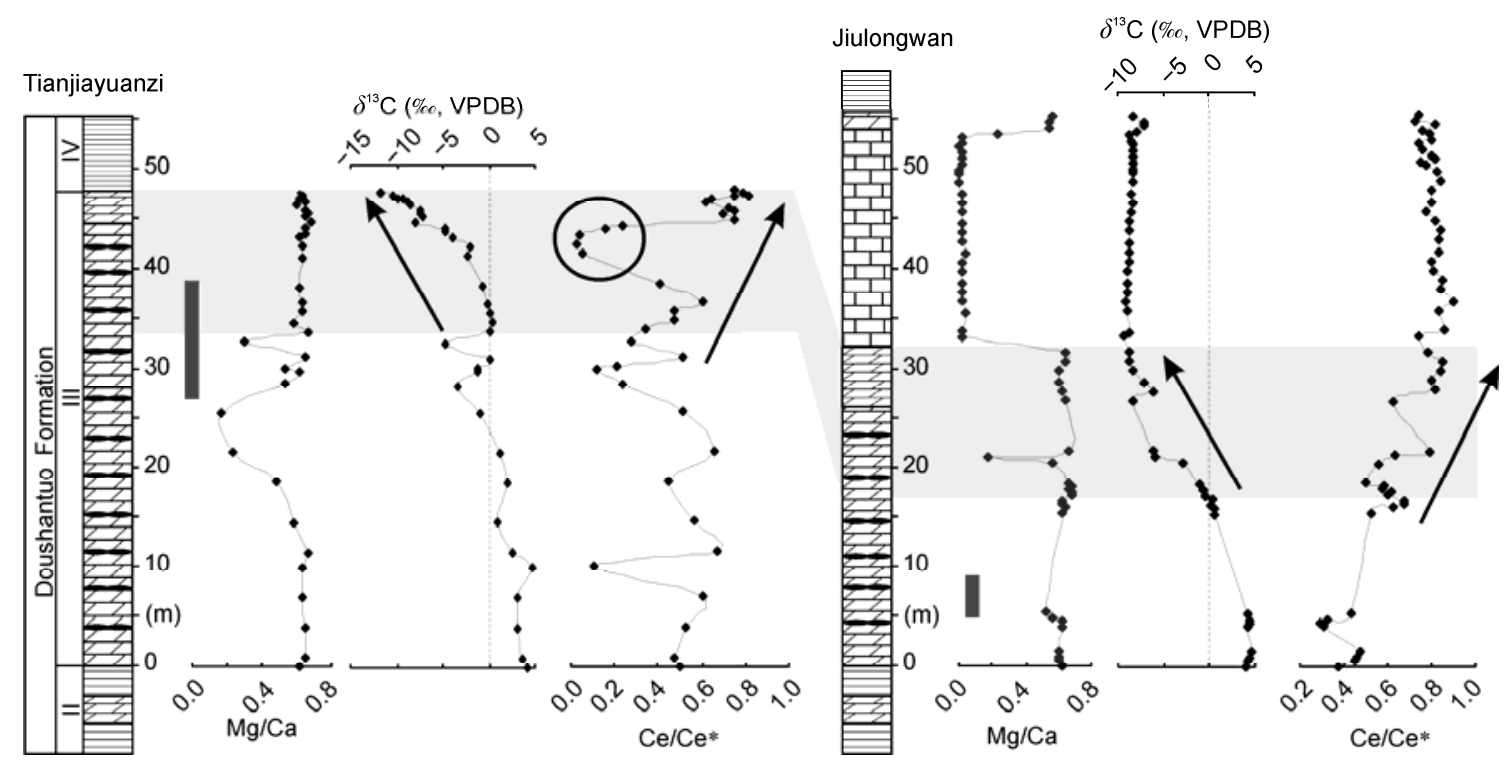

Figure $2 \mathrm{Mg} / \mathrm{Ca}$ ratio, $\delta^{13} \mathrm{C}_{\text {carb }}$ and $\mathrm{Ce} / \mathrm{Ce}^{*}$ profiles of Member III at the Tianjiayuanzi and Jiulongwan sections. The limestone unit is absent at Tianjiayuanzi. Shaded band represents proposed correlation between the two sections. Vertical bars represent the stratigraphic ranges of Ediacaran acanthomorphs. Acanthomorph occurrence data for Jiulongwan section are from Yin et al. [19]. See Figure 1 for lithologic legends. 
Table 1 Analytical data for member III carbonates of the Doushantuo Formation at Jiulongwan (prefix 05HND) and Tianjiayuanzi (prefix 08TJYZ) (D, dolostone; DL, dolomitic limestone)

\begin{tabular}{|c|c|c|c|c|c|c|c|c|c|c|c|c|c|c|c|}
\hline Sample & Lithology & $\begin{array}{c}\text { Hight } \\
(\mathrm{m})\end{array}$ & $\begin{array}{c}\delta^{13} \mathrm{C} \\
(\%, \mathrm{VPDB})\end{array}$ & $\begin{array}{c}\delta^{18} \mathrm{O} \\
(\%, \mathrm{VPDB})\end{array}$ & $\begin{array}{l}\sum R E E \\
(\mathrm{ppm})\end{array}$ & $\mathrm{Y} / \mathrm{Ho}$ & $\mathrm{Ce} / \mathrm{Ce}^{*}$ & $\mathrm{Pr} / \mathrm{Pr}^{*}$ & $\mathrm{Nd}_{\mathrm{SN}} / \mathrm{Yb}_{\mathrm{SN}}$ & ${ }^{87} \mathrm{Sr} /{ }^{86} \mathrm{Sr}$ & $\mathrm{Mg} / \mathrm{Ca}$ & $\mathrm{Mn}$ & $\mathrm{Sr}$ & $\mathrm{Zr}$ & $\mathrm{Mn} / \mathrm{Sr}$ \\
\hline 05HND-26 & $\mathrm{D}$ & 0.0 & 4.1 & -2.8 & 5.679 & 32.6 & 0.37 & 1.43 & 1.26 & & 0.63 & 89.147 & 121.146 & 0.004 & 0.74 \\
\hline 05HND-27 & D & 0.5 & 4.4 & -3.5 & 9.299 & 29.4 & 0.45 & 1.25 & 2.46 & 0.708035 & 0.61 & 60.207 & 121.035 & 0.001 & 0.50 \\
\hline 05HND-28 & D & 1.0 & 4.5 & -4.0 & 10.019 & 33.3 & 0.46 & 1.29 & 3.60 & & 0.60 & 72.358 & 122.030 & 0.010 & 0.59 \\
\hline 05HND-29 & D & 1.5 & 4.6 & -4.3 & 11.295 & 26.3 & 0.48 & 1.32 & 2.61 & & 0.61 & 75.649 & 113.373 & 0.004 & 0.67 \\
\hline 05HND-30 & D & 4.0 & 4.4 & -4.5 & 10.742 & 31.6 & 0.31 & 1.35 & 2.82 & 0.708328 & 0.63 & 70.269 & 72.927 & 0.000 & 0.96 \\
\hline 05HND-31 & D & 4.5 & 4.5 & -4.2 & 7.247 & 32.3 & 0.29 & 1.47 & 2.50 & & 0.62 & 49.086 & 57.173 & 0.000 & 0.86 \\
\hline 05HND-32 & D & 4.8 & 4.5 & -4.1 & 6.093 & 34.0 & 0.33 & 1.26 & 2.54 & & 0.57 & 54.980 & 78.630 & 0.000 & 0.70 \\
\hline 05HND-33 & D & 5.3 & 4.2 & -6.2 & 11.443 & 33.3 & 0.43 & 1.25 & 2.79 & 0.709348 & 0.52 & 54.949 & 113.528 & 0.002 & 0.48 \\
\hline 05HND-34 & D & 15.3 & 0.6 & -4.6 & 8.764 & 33.0 & 0.52 & 1.23 & 1.11 & & 0.62 & 111.902 & 34.064 & 0.004 & 3.29 \\
\hline 05HND-35 & D & 15.9 & 0.6 & -5.6 & 18.430 & 26.8 & 0.62 & 1.19 & 1.07 & & 0.65 & 116.202 & 38.145 & 0.002 & 3.05 \\
\hline 05HND-36 & D & 16.3 & 0.2 & -3.8 & 16.336 & 29.5 & 0.67 & 1.20 & 0.83 & 0.709111 & 0.63 & 117.407 & 40.100 & 0.010 & 2.93 \\
\hline 05HND-37 & D & 16.7 & 0.5 & -1.6 & 14.691 & 28.2 & 0.67 & 1.13 & 0.62 & & 0.63 & 139.209 & 45.067 & 0.032 & 3.09 \\
\hline 05HND-38 & D & 17.2 & -0.4 & -4.0 & 12.479 & 28.1 & 0.60 & 1.30 & 0.83 & & 0.68 & 124.991 & 35.100 & 0.026 & 3.56 \\
\hline 05HND-39 & D & 17.5 & -0.4 & -3.3 & 12.340 & 25.6 & 0.61 & 1.28 & 0.79 & 0.708701 & 0.68 & 127.254 & 37.257 & 0.045 & 3.42 \\
\hline 05HND-40 & D & 17.8 & -0.7 & -5.0 & 12.093 & 26.5 & 0.57 & 1.22 & 0.92 & & 0.66 & 111.125 & 31.868 & 0.011 & 3.49 \\
\hline 05HND-41 & D & 18.1 & -0.8 & -5.1 & 10.720 & 29.7 & 0.59 & 1.20 & 1.16 & & 0.69 & 101.111 & 31.323 & 0.007 & 3.23 \\
\hline 05HND-42 & D & 18.4 & -1.0 & -5.0 & 8.900 & 30.7 & 0.50 & 1.31 & 0.95 & 0.708758 & 0.67 & 106.978 & 30.871 & 0.007 & 3.47 \\
\hline 05HND-43 & D & 20.4 & -2.9 & -6.2 & 11.507 & 27.8 & 0.56 & 1.26 & 0.83 & & 0.56 & 207.811 & 48.422 & 0.046 & 4.29 \\
\hline 05HND-44 & DL & 21.1 & -5.9 & -10.6 & 9.458 & 40.6 & 0.64 & 1.15 & 1.27 & & 0.18 & 283.149 & 82.245 & 0.022 & 3.44 \\
\hline 05HND-45 & $\mathrm{D}$ & 21.7 & -6.2 & -6.1 & 9.529 & 32.2 & 0.79 & 1.20 & 1.23 & 0.708538 & 0.67 & 139.800 & 40.299 & 0.010 & 3.47 \\
\hline 05HND-46 & D & 26.7 & -8.3 & -5.9 & 6.718 & 25.9 & 0.62 & 1.22 & 0.99 & & 0.65 & 91.193 & 34.751 & 0.007 & 2.62 \\
\hline 05HND-47 & D & 27.7 & -6.0 & -3.7 & 27.252 & 26.6 & 0.81 & 1.08 & 1.27 & & 0.63 & 164.536 & 49.662 & 0.212 & 3.31 \\
\hline 05HND-48 & D & 28.7 & -7.2 & -5.0 & 24.613 & 25.6 & 0.80 & 1.12 & 1.54 & 0.709277 & 0.60 & 129.873 & 46.059 & 0.015 & 2.82 \\
\hline 05HND-49 & D & 29.7 & -8.4 & -6.8 & 29.471 & 26.5 & 0.84 & 1.09 & 1.38 & & 0.60 & 199.604 & 41.585 & 0.031 & 4.80 \\
\hline 05HND-50 & D & 30.7 & -8.7 & -7.1 & 33.797 & 28.8 & 0.85 & 1.09 & 1.63 & & 0.64 & 187.446 & 33.578 & 0.007 & 5.58 \\
\hline 05HND-51 & D & 31.7 & -8.9 & -7.4 & 28.363 & 25.6 & 0.78 & 1.12 & 1.26 & 0.709973 & 0.64 & 197.038 & 33.788 & 0.003 & 5.83 \\
\hline 05HND-52 & $\mathrm{DL}$ & 33.2 & -9.3 & -10.3 & 27.474 & 29.4 & 0.74 & 1.16 & 1.39 & & 0.02 & 61.602 & 160.451 & 0.015 & 0.38 \\
\hline 05HND-53 & DL & 33.7 & -8.7 & -10.3 & 32.351 & 27.3 & 0.86 & 1.07 & 1.42 & & 0.03 & 86.051 & 150.473 & 0.012 & 0.57 \\
\hline 05HND-54 & DL & 35.7 & -8.9 & -9.5 & 40.815 & 25.3 & 0.83 & 1.13 & 1.49 & 0.70916 & 0.04 & 82.571 & 169.326 & 0.023 & 0.49 \\
\hline 05HND-55 & DL & 36.7 & -9.1 & -9.7 & 33.442 & 25.1 & 0.90 & 1.06 & 1.71 & & 0.02 & 72.593 & 148.165 & 0.011 & 0.49 \\
\hline 05HND-56 & DL & 37.7 & -9.1 & -9.7 & 32.375 & 27.0 & 0.84 & 1.13 & 1.66 & & 0.02 & 56.580 & 162.040 & 0.000 & 0.35 \\
\hline 05HND-57 & DL & 38.7 & -9.0 & -9.4 & 29.418 & 26.4 & 0.85 & 1.11 & 1.64 & 0.709014 & 0.02 & 68.625 & 173.566 & 0.035 & 0.40 \\
\hline 05HND-58 & DL & 39.7 & -8.9 & -9.3 & 28.672 & 27.9 & 0.81 & 1.12 & 1.63 & & 0.01 & 52.444 & 172.148 & 0.010 & 0.30 \\
\hline 05HND-59 & DL & 40.7 & -8.9 & -9.2 & 28.085 & 26.4 & 0.80 & 1.14 & 1.46 & & 0.01 & 52.793 & 183.839 & 0.008 & 0.29 \\
\hline 05HND-60 & DL & 41.7 & -8.8 & -8.9 & 32.920 & 27.5 & 0.84 & 1.13 & 1.77 & 0.708959 & 0.04 & 68.273 & 152.571 & 0.005 & 0.45 \\
\hline 05HND-61 & DL & 42.7 & -8.7 & -8.8 & 26.294 & 28.7 & 0.83 & 1.13 & 1.54 & & 0.02 & 51.578 & 161.711 & 0.039 & 0.32 \\
\hline 05HND-62 & DL & 43.7 & -8.8 & -8.9 & 40.064 & 28.3 & 0.85 & 1.05 & 1.41 & & 0.02 & 49.313 & 178.642 & 0.003 & 0.28 \\
\hline 05HND-63 & DL & 44.7 & -8.8 & -8.7 & 30.617 & 28.5 & 0.81 & 1.11 & 1.61 & 0.708942 & 0.01 & 40.933 & 195.144 & 0.013 & 0.21 \\
\hline 05HND-64 & DL & 45.7 & -8.6 & -8.5 & 19.036 & 30.3 & 0.78 & 1.18 & 1.40 & & 0.01 & 47.724 & 185.848 & 0.011 & 0.26 \\
\hline 05HND-65 & DL & 46.7 & -8.4 & -8.1 & 15.807 & 31.3 & 0.80 & 1.09 & 1.33 & & 0.01 & 41.063 & 166.550 & 0.012 & 0.25 \\
\hline 05HND-66 & DL & 47.7 & -8.6 & -8.2 & 33.064 & 30.3 & 0.80 & 1.11 & 1.17 & 0.709051 & 0.01 & 55.352 & 217.869 & 0.016 & 0.25 \\
\hline 05HND-67 & DL & 48.7 & -8.4 & -7.9 & 20.576 & 32.2 & 0.84 & 1.08 & 1.16 & & 0.01 & 47.989 & 191.855 & 0.008 & 0.25 \\
\hline 05HND-68 & DL & 49.7 & -8.4 & -7.8 & 17.956 & 28.3 & 0.83 & 1.07 & 1.43 & & 0.01 & 42.106 & 148.899 & 0.002 & 0.28 \\
\hline 05HND-69 & DL & 50.2 & -8.4 & -7.4 & 16.633 & 30.0 & 0.77 & 1.15 & 1.34 & 0.709042 & 0.01 & 37.255 & 163.663 & 0.004 & 0.23 \\
\hline 05HND-70 & DL & 50.7 & -8.2 & -7.3 & 15.101 & 29.0 & 0.75 & 1.22 & 1.39 & & 0.01 & 34.100 & 149.678 & 0.013 & 0.23 \\
\hline 05HND-15 & $\mathrm{DL}$ & 50.8 & -8.3 & -7.3 & 13.287 & 27.0 & 0.81 & 1.12 & 1.07 & & 0.01 & 39.028 & 121.316 & 0.016 & 0.32 \\
\hline 05HND-14 & DL & 51.3 & -8.4 & -7.1 & 15.047 & 27.9 & 0.80 & 1.14 & 1.13 & 0.708919 & 0.02 & 40.871 & 122.498 & 0.002 & 0.33 \\
\hline 05HND-13 & $\mathrm{DL}$ & 51.8 & -8.4 & -7.3 & 11.220 & 31.9 & 0.76 & 1.14 & 1.32 & & 0.01 & 47.288 & 119.106 & 0.005 & 0.40 \\
\hline
\end{tabular}


(Continued)

\begin{tabular}{|c|c|c|c|c|c|c|c|c|c|c|c|c|c|c|c|}
\hline Sample & Lithology & $\begin{array}{l}\text { Hight } \\
(\mathrm{m})\end{array}$ & $\begin{array}{c}\delta^{13} \mathrm{C} \\
(\%, \mathrm{VPDB})\end{array}$ & $\begin{array}{c}\delta^{18} \mathrm{O} \\
(\% o, \mathrm{VPDB})\end{array}$ & $\begin{array}{l}\sum \mathrm{REE} \\
(\mathrm{ppm})\end{array}$ & Y/Ho & $\mathrm{Ce} / \mathrm{Ce}^{*}$ & $\mathrm{Pr} / \mathrm{Pr}^{*}$ & $\mathrm{Nd}_{\mathrm{SN}} / \mathrm{Yb}_{\mathrm{SN}}$ & ${ }^{87} \mathrm{Sr} /{ }^{86} \mathrm{Sr}$ & $\mathrm{Mg} / \mathrm{Ca}$ & $\mathrm{Mn}$ & $\mathrm{Sr}$ & $\mathrm{Zr}$ & $\mathrm{Mn} / \mathrm{Sr}$ \\
\hline 05HND-12 & DL & 52.4 & -8.4 & -7.3 & 10.129 & 29.3 & 0.74 & 1.14 & 1.05 & & 0.01 & 39.661 & 134.851 & 0.011 & 0.29 \\
\hline 05HND-11 & DL & 52.9 & -8.5 & -7.4 & 14.393 & 31.0 & 0.80 & 1.11 & 1.11 & 0.708905 & 0.02 & 57.109 & 133.113 & 0.007 & 0.43 \\
\hline 05HND-10 & DL & 53.4 & -8.8 & -8.1 & 16.052 & 26.8 & 0.79 & 1.18 & 1.33 & & 0.02 & 49.165 & 127.732 & 0.006 & 0.38 \\
\hline 05HND-9 & DL & 53.8 & -8.0 & -5.1 & 23.680 & 25.4 & 0.76 & 1.17 & 1.11 & & 0.23 & 93.246 & 107.805 & 0.021 & 0.86 \\
\hline 05HND-8 & $\mathrm{D}$ & 54.3 & -7.1 & -2.3 & 20.442 & 27.6 & 0.81 & 1.04 & 1.03 & 0.70924 & 0.55 & 152.334 & 116.572 & 0.030 & 1.31 \\
\hline 05HND-6 & $\mathrm{D}$ & 55.4 & -8.4 & -2.1 & 24.547 & 29.7 & 0.74 & 1.07 & 0.69 & & 0.57 & 290.823 & 221.293 & 0.038 & 1.31 \\
\hline 08TJYZ-1 & $\mathrm{D}$ & 47.5 & -11.9 & -5.0 & 10.900 & 31.8 & 0.75 & 1.10 & 0.92 & 0.709212 & 0.62 & 199.202 & 61.658 & 0.266 & 3.23 \\
\hline 08TJYZ-2 & $\mathrm{D}$ & 47.3 & -10.5 & -5.4 & 13.277 & 29.0 & 0.79 & 1.08 & 1.00 & & 0.64 & 145.773 & 40.065 & 0.101 & 3.64 \\
\hline 08TJYZ-3 & $\mathrm{D}$ & 47.1 & -9.9 & -5.2 & 11.385 & 33.6 & 0.76 & 1.07 & 0.97 & & 0.62 & 116.385 & 51.454 & 0.119 & 2.26 \\
\hline 08TJYZ-4 & $\mathrm{D}$ & 46.9 & -9.4 & -4.5 & 8.301 & 25.7 & 0.82 & 1.03 & 0.68 & 0.708888 & 0.61 & 133.155 & 47.133 & 0.083 & 2.83 \\
\hline 08TJYZ-5 & $\mathrm{D}$ & 46.6 & -8.7 & -4.1 & 11.252 & 27.9 & 0.64 & 1.23 & 0.92 & & 0.66 & 93.771 & 37.440 & 0.069 & 2.50 \\
\hline 08TJYZ-7 & $\mathrm{D}$ & 45.8 & -7.5 & -2.2 & 13.459 & 30.1 & 0.72 & 1.22 & 1.11 & 0.709034 & 0.65 & 113.543 & 47.131 & 0.062 & 2.41 \\
\hline 08TJYZ-8 & $\mathrm{D}$ & 45.5 & -7.4 & -2.2 & 17.512 & 29.9 & 0.75 & 1.09 & 1.31 & & 0.66 & 120.525 & 37.392 & 0.052 & 3.22 \\
\hline 08TJYZ-9 & $\mathrm{D}$ & 45.15 & -7.2 & -2.8 & 15.765 & 33.0 & 0.70 & 1.30 & 0.94 & & 0.66 & 146.253 & 37.740 & 0.042 & 3.88 \\
\hline 08TJYZ-10 & $\mathrm{D}$ & 44.5 & -7.8 & -5.3 & 7.093 & 26.8 & 0.75 & 1.25 & 0.79 & 0.70868 & 0.68 & 212.049 & 50.519 & 0.016 & 4.20 \\
\hline 08TJYZ-11 & $\mathrm{D}$ & 44.0 & -4.6 & -5.2 & 1.123 & 37.8 & 0.24 & 1.65 & 1.05 & & 0.66 & 84.614 & 71.435 & 0.057 & 1.18 \\
\hline 08TJYZ-12 & $\mathrm{D}$ & 43.5 & -4.7 & -6.1 & 1.256 & 38.1 & 0.15 & 1.64 & 0.83 & & 0.65 & 61.880 & 97.039 & 0.039 & 0.64 \\
\hline 08TJYZ-13 & $\mathrm{D}$ & 43.15 & -4.0 & -6.2 & 1.148 & 35.5 & 0.04 & 1.49 & 1.21 & 0.708354 & 0.61 & 45.833 & 108.583 & 0.018 & 0.42 \\
\hline 08TJYZ-14 & $\mathrm{D}$ & 42.1 & -2.1 & -5.6 & 0.939 & 40.7 & 0.02 & 1.36 & 1.51 & & 0.64 & 47.407 & 67.292 & 0.030 & 0.70 \\
\hline 08TJYZ-15 & $\mathrm{D}$ & 41.1 & -2.2 & -6.1 & 1.082 & 40.0 & 0.05 & 1.77 & 0.95 & 0.709446 & 0.63 & 60.657 & 40.744 & 0.011 & 1.49 \\
\hline 08TJYZ-18 & $\mathrm{D}$ & 38.1 & -0.7 & -3.4 & 3.386 & 38.1 & 0.41 & 1.20 & 1.15 & & 0.62 & 32.723 & 51.359 & 0.024 & 0.64 \\
\hline 08TJYZ-21 & $\mathrm{D}$ & 36.5 & -0.2 & -3.4 & 7.553 & 44.0 & 0.61 & 1.11 & 1.02 & & 0.63 & 25.376 & 64.647 & 0.035 & 0.39 \\
\hline 08TJYZ-22 & $\mathrm{D}$ & 35.5 & 0.1 & -3.1 & 5.724 & 29.2 & 0.47 & 1.27 & 0.94 & 0.709207 & 0.63 & 35.006 & 49.441 & 0.018 & 0.71 \\
\hline 08TJYZ-24 & $\mathrm{D}$ & 33.5 & 0.0 & -4.8 & 2.189 & 28.9 & 0.34 & 1.45 & 0.67 & & 0.67 & 36.527 & 37.088 & 0.017 & 0.98 \\
\hline 08TJYZ-25 & $\mathrm{D}$ & 32.5 & -4.6 & -6.4 & 3.197 & 61.3 & 0.28 & 1.97 & 0.47 & 0.708475 & 0.30 & 27.919 & 35.917 & 0.065 & 0.78 \\
\hline 08TJYZ-27 & $\mathrm{D}$ & 31.0 & 0.1 & -4.7 & 3.009 & 35.1 & 0.52 & 1.21 & 0.60 & & 0.65 & 43.258 & 43.513 & 0.028 & 0.99 \\
\hline 08TJYZ-28 & $\mathrm{D}$ & 30.0 & -1.2 & -5.0 & 5.058 & 47.4 & 0.21 & 1.46 & 1.31 & & 0.53 & 32.737 & 80.115 & 0.338 & 0.41 \\
\hline 08TJYZ-35 & $\mathrm{D}$ & 29.7 & -1.3 & -2.3 & 1.589 & 39.0 & 0.12 & 1.50 & 0.68 & & 0.62 & 45.630 & 216.814 & 0.030 & 0.21 \\
\hline 08TJYZ-36 & $\mathrm{D}$ & 28.3 & -3.5 & -2.8 & 1.940 & 35.8 & 0.24 & 1.58 & 0.40 & & 0.54 & 44.972 & 184.200 & 0.032 & 0.24 \\
\hline 08TJYZ-37 & D & 25.5 & -1.0 & -5.8 & 5.964 & 38.2 & 0.52 & 1.19 & 1.24 & 0.708637 & 0.16 & 24.566 & 235.700 & 0.046 & 0.10 \\
\hline 08TJYZ-38 & $\mathrm{D}$ & 21.5 & 1.2 & -3.5 & 11.036 & 33.1 & 0.66 & 1.13 & 1.45 & & 0.23 & 33.484 & 263.745 & 0.048 & 0.13 \\
\hline 08TJYZ-39 & $\mathrm{D}$ & 18.5 & 2.0 & -4.2 & 11.046 & 39.2 & 0.44 & 1.26 & 1.12 & & 0.48 & 37.708 & 293.655 & 0.046 & 0.13 \\
\hline 08TJYZ-40 & $\mathrm{D}$ & 14.5 & 1.0 & -5.7 & 5.445 & 34.7 & 0.57 & 1.15 & 1.38 & 0.708635 & 0.58 & 43.411 & 62.447 & 0.014 & 0.70 \\
\hline 08TJYZ-41 & $\mathrm{D}$ & 11.5 & 2.6 & -6.0 & 6.959 & 30.3 & 0.67 & 1.15 & 1.39 & & 0.67 & 62.470 & 38.199 & 0.027 & 1.64 \\
\hline 08TJYZ-42 & D & 10.0 & 4.7 & -6.2 & 1.183 & 39.7 & 0.10 & 1.59 & 1.47 & & 0.63 & 43.482 & 43.168 & 0.010 & 1.01 \\
\hline 08TJYZ-43 & $\mathrm{D}$ & 7.0 & 3.0 & -6.0 & 14.818 & 31.5 & 0.61 & 1.20 & 1.72 & 0.708777 & 0.64 & 84.289 & 37.655 & 0.051 & 2.24 \\
\hline 08TJYZ-44 & $\mathrm{D}$ & 4.0 & 3.0 & -6.0 & 12.280 & 30.4 & 0.52 & 1.27 & 1.57 & & 0.65 & 87.796 & 39.862 & 0.016 & 2.20 \\
\hline 08TJYZ-45 & $\mathrm{D}$ & 1.0 & 3.6 & -5.2 & 12.127 & 34.5 & 0.47 & 1.26 & 2.72 & & 0.65 & 101.784 & 55.490 & 0.014 & 1.83 \\
\hline 08TJYZ-46 & $\mathrm{D}$ & 0.0 & 4.2 & -4.4 & 14.934 & 36.4 & 0.50 & 1.23 & 2.01 & 0.708475 & 0.61 & 109.152 & 87.835 & 0.014 & 1.24 \\
\hline
\end{tabular}

$\mathrm{Zr}$ concentrations and $\sum \mathrm{REE}$, and $\mathrm{Zr}$ concentrations and $\mathrm{Ce} / \mathrm{Ce}^{*}$ values of the studied samples (Figure S4) [17]. A comparative study using different acids $(0.5 \mathrm{~N}$ acetic acid, $3.4 \mathrm{~N}$ acetic acid, and $\mathrm{HF}+\mathrm{HNO}_{3}+\mathrm{HClO}_{4}$ ) has demonstrated that carbonate dissolved in dilute acetic acid is nearly free of detritus contamination [21]. Similarly, different acid treatments of Jiulongwan samples using $0.5 \mathrm{~N}$ and $1.0 \mathrm{~N}$ acetic acids gave similar REE distribution patterns. Though $\mathrm{Ce} / \mathrm{Ce}^{*}$ values and $\Sigma \mathrm{REE}$ with $0.5 \mathrm{~N}$ acetic acid treatment are less than those with $1.0 \mathrm{~N}$ acetic acid treatment, results from the two treatments are positively correlated (Figure S5). The comparative study therefore indicates the conservative nature of REE as a group and particularly the stratigraphic pattern of $\mathrm{Ce} / \mathrm{Ce}^{*}$ values can provide useful 
information about redox changes in the water column, as demonstrated in several geochemical studies of the Black Sea [22] and the geological record [23].

The $\mathrm{Ce} / \mathrm{Ce}^{*}$ shifts are not a mathematical artifact of anomalously high La concentrations (Figure S3). Ce/Ce* vs. Pr/Pr* plots [24], where our data mostly plot in the field IIIb (Figure S6), show that negative Ce anomalies are not caused by anomalous La concentrations, but reflect true negative Ce anomalies. Diagenesis (e.g. early dolomitization) cannot explain the $\delta^{13} \mathrm{C}_{\text {carb }}$ and $\mathrm{Ce} / \mathrm{Ce}^{*}$ shifts either. Most of the samples have $\delta^{18} \mathrm{O}>-10 \%$ and $\mathrm{Mn} / \mathrm{Sr}<2$ (Table 1), suggesting little diagenetic alteration. ${ }^{87} \mathrm{Sr} /{ }^{86} \mathrm{Sr}$ ratios vary from 0.708035 to 0.709973 (average 0.708961 ) for samples from Jiulongwan, and from 0.708354 to 0.709446 (average= 0.708821 ) for samples from Tianjiayuanzi (Table 1). The ${ }^{87} \mathrm{Sr} /{ }^{86} \mathrm{Sr}$ data from both sections are consistent with those for drillhole samples of the same horizon in the Yangtze Gorges area [25], and with the secular change in the ${ }^{87} \mathrm{Sr} /{ }^{86} \mathrm{Sr}$ ratio of Ediacaran seawater [26]. Importantly, the $\delta^{13} \mathrm{C}_{\text {carb }}$ and $\mathrm{Ce} / \mathrm{Ce}^{*}$ shifts are independent of lithological changes, occurring in the dolostone unit and not coinciding with a lithological shift from dolostone to limestone at Jiulongwan. This lithological observation is further confirmed by $\mathrm{Mg} / \mathrm{Ca}$ ratio data. At Tianjiayuanzi, $\delta^{13} \mathrm{C}_{\mathrm{carb}}$ changes from $4.7 \%$ o to $-11.9 \%$ and $\mathrm{Ce} / \mathrm{Ce}^{*}$ vary between 0.02 and 0.82 , while $\mathrm{Mg} / \mathrm{Ca}$ ratios remain relatively stable around $0.59(N=35, \mathrm{SD}=0.12)$. Similarly, at Jiulongwan, $\delta^{13} \mathrm{C}_{\text {carb }}$ changes from $4.6 \%$ o to $-8.9 \%$ and $\mathrm{Ce} / \mathrm{Ce} *$ from 0.29 to 0.85 in Member III dolostone unit, while $\mathrm{Mg} / \mathrm{Ca}$ ratios remain stable around $0.61(N=26, \mathrm{SD}=0.10)$ (Figure 2).

The REE distribution patterns of the Doushantuo Formation show features (e.g. flat NASC-normalized REE patterns and low Y/Ho ratios) somewhat different from normal seawater REE patterns, which are characterized by HREE enrichment and elevated Y/Ho ratios [27]. For example, Doushantuo Y/Ho ratios are 25.1-40.6 (average 28.9) and 25.7-61.3 (average 35.4) at Jiulongwan and Tianjiayuanzi, respectively (Table 1), lower than typical marine values (60-90) but similar to average upper continental crust and to freshwater carbonates from the Green River Formation [28]. As colloidal phases of river waters generally show LREE enriched patterns whereas filtered river water is HREE-enriched [29], the flat REE distribution patterns and lower Y/Ho ratios of Doushantuo carbonates may imply the incorporation of near-shore colloids during their precipitation in a shallow coastal environment, an interpretation that has also been used to explain the relatively unfractionated shale-normalized REE+Y patterns of most Neoproterozoic carbonates from southwestern and central Africa [30].

\subsection{Shallow marine anoxia}

There have been numerous attempts to reconstruct the redox history in Ediacaran deep oceans. Fe speciation data from well-preserved Neoproterozoic sedimentary rocks suggest an anoxic ferruginous condition as a general feature of deep oceans between 750 and $540 \mathrm{Ma}$ [31]. Fe speciation data of the Ediacaran-early Cambrian sedimentary rocks (Doushantuo, Liuchapo, and Niutitang formations) deposited in outer shelf to basinal environment in South China suggest an anoxic condition in deep oceans [31-33], although paleontological data indicate that the largely anoxic basin may be punctuated by brief oxic episodes [34,35].

Whereas anoxic conditions appear to have been persistent in the deep basin, several lines of evidence suggest that marine shelves on the Yangtze platform may have experienced regionally variable redox conditions after the Marinoan glaciation. Iron speciation data $\left(\mathrm{Fe}_{\mathrm{HR}} / \mathrm{Fe}_{\mathrm{T}}\right.$ and $\mathrm{Fe}_{\mathrm{py}} /$ $\mathrm{Fe}_{\mathrm{HR}}$ ) show an anoxic to euxinic environment for the Member II black shale samples of the early Ediacaran Doushantuo Formation in the Yangtze Gorges area [32], whereas DOP (degree of pyritization) measurements for the same black shales indicate variable redox conditions, fluctuating between oxic, dysoxic, and euxinic environments [9], the discrepancy was partly due to the application of different proxies $\left(\mathrm{Fe}_{\mathrm{py}} / \mathrm{Fe}_{\mathrm{HR}}\right.$ vs. DOP). Iron speciation proxies, however, are not readily applicable to carbonate successions such as Member III, and this is the main drive for us to turn to REE proxies. Based on empirical studies of biogenic apatite, Wright et al. [36] defined $\mathrm{Ce} / \mathrm{Ce}^{*}=0.8$ as a cut-off between anoxic and oxic conditions. This has been previously applied in the study of Doushantuo phosphorites at Weng'an in Guizhou Province, where bottom water conditions changed from anoxic in the lower part $\left(\mathrm{Ce} / \mathrm{Ce}^{*}\right.$ from 0.84 to 0.91 , averaging 0.88$)$ to oxic in the upper part $(\mathrm{Ce} /$ Ce* values range from 0.45 to 0.70 , averging 0.55 ) of the Doushantuo Formation [37].

The high-resolution data presented here show a pronounced negative $\delta^{13} \mathrm{C}_{\text {carb }}$ excursion (EN3) and synchronous positive $\mathrm{Ce} / \mathrm{Ce}^{*}$ shift in Member III of the Ediacaran Doushantuo Formation in the Yangtze Gorges area. Comparison of the $\mathrm{Ce} / \mathrm{Ce}^{*}$ profiles at Tianjiayuanzi and Jiulongwan shows that the former exhibits a greater degree of fluctuation, which may be related to the relatively shallower environment at Tianjiayuanzi which is more susceptible to local variations. Indeed, the low $\mathrm{Ce} / \mathrm{Ce}^{*}$ values at 41-44 $\mathrm{m}$ at Tianjiayuanzi coincide with a lithological interval characterized by oncolitic lenses and cross-bedding structures (Figure S2d-f) that may reflect deposition in a locally very shallow oxic environment. Ignoring these data (circled in Figure 2), the positive $\mathrm{Ce} / \mathrm{Ce} *$ shift in association with negative $\delta^{13} \mathrm{C}_{\text {carb }}$ trend (shaded in Figure 2) are prominent at both sections. The shifts in $\delta^{13} \mathrm{C}_{\text {carb }}$ and $\mathrm{Ce} / \mathrm{Ce} *$ likely reflect a change from oxic to suboxic-anoxic in shallow waters, resulted from the upwelling of anoxic deep water, which also introduced ${ }^{12} \mathrm{C}$-rich DOC and DIC to the inner shelf of the Yangtze Gorges area.

Integrated with previously published data, our new data suggested the following scenario of redox change during the deposition of lower-middle Doushantuo Formation in the 
Yangtze Gorges area. During the early Doushantuo time (Member II), the shallow shelf environment may have been largely anoxic, punctuated by brief oxic episodes. But this was succeeded by oxic conditions during the deposition of the lower Member III dolostone. The shallow shelf returned to suboxic or anoxic conditions during the deposition of the upper part of Member III (uppermost dolostone unit and limestone unit).

Considering that the upper part of Member III records a slightly deepening-upward sequence, it is possible that transgression might have initiated oceanic upwelling and brought the redox-cline to the inner shelf, leading to shallow marine anoxia. Although the exact duration of this event is currently unknown, this shallow marine anoxia may represent at least a regional event in the Yangtze Gorges area because $\delta^{13} \mathrm{C}_{\text {carb }}$ negative excursions equivalent to EN3 have been reported from many sections in this area [14]. It is possible that shallow marine anoxia may have been widespread beyond the Yangtze Gorges area during the middle Ediacaran Period, if other $\delta^{13} \mathrm{C}_{\text {carb }}$ negative excursions equivalent to EN3 [7,38-40] have similar origins.

\subsection{Anoxia and the decline of Doushantuo acantho- morphs}

Ediacaran acanthomorphs represent a unique assemblage of large and morphologically complex acritarchs that occur in the lower-middle Ediacaran successions in South China and elsewhere in the world [3]. Ediacaran acanthomorphs have long been regarded as the resting cysts of various unicellular phytoplanktonic algae [41]. However, recent studies have shown that they may have more diverse affinities, and some of them may be animal resting cysts [6,42].

Several hypotheses have been proposed to explain the abrupt disappearance of Ediacaran acanthomorphs before the appearance of classical Ediacara megafossils in the middle Ediacaran time [3]. Cohen et al. [6] interpreted the large Ediacaran acanthomprphs as animal resting cysts, and proposed that their evolutionary rise in the early Ediacaran Period was an ecological response to bottom water anoxia, and their disappearance in the late Ediacaran Period was due to the pervasive oxygenation of continental shelves. Whether Ediacaran acanthomorphs are dormant cysts of protists or animals, it is clear that they must have spent at least part of their life cycle resting in the benthic realm [43]. Thus, their ecology must have been affected by redox conditions in the benthic environments. If our inference of a widespread middle Ediacaran shallow marine anoxia event is correct, this event is expected to have had an impact on the ecological environment of Ediacaran acanthomorphs. Given the temporal coincidence between the inferred anoxia and the disappearance of Ediacaran acanthomorphs in Member III, it is possible that this middle Ediacaran shallow marine anoxia event may have been the cause for the decline (rather than rise) of the Doushantuo acanthomorphs.
This hypothesis is consistent with emerging data from the Patom Complex in central Siberia, where the Ura Formation that bears Ediacaran acanthomorphs is stratigraphically below the Zhuya Group that is characterized by a $-10 \%$ o $\delta^{13} \mathrm{C}_{\text {carb }}$ excursion which is a potential equivalent to EN3 and the Shuram excursion $[44,45]$.

\section{Conclusions}

Middle Ediacaran shallow marine anoxia recorded by the pronounced negative $\delta^{13} \mathrm{C}_{\text {carb }}$ shift and REE distribution patterns resembles anoxia events at the Permian-Triassic and Ediacaran-Cambrian transitions; these anoxia events have been interpreted as evidence for oceanic overturn or expansion of anoxic deep water masses onto shallow shelves, causing oxygen depletion and negative $\delta^{13} \mathrm{C}$ shifts in shallow environments $[46,47]$. Both anoxia events at the Permian-Triassic and Ediacaran-Cambrian transitions caused major extinctions. We propose that the middle Ediacaran anoxia event also had similar geobiological consequences: it may have driven the decline of Ediacaran acanthomorphs, at least in the Yangtze Gorges area.

This work was supported by the Knowledge Innovation Program of Chinese Academy of Sciences (KZCX2-EW-119), the Ministry of Science and Technology of China (2011CB808805), the National Natural Science Foundation of China (41028002, 40872026 and 41130209), and Ministry of Education (306007). We thank Chen Xiaoming and Yang Tao for analytical assistance. Linda Kah and Graham Shields provided constructive criticism on an earlier draft of the paper.

1 Fike D A, Grotzinger J P, Pratt L M, et al. Oxidation of the Ediacaran ocean. Nature, 2006, 444: 744-747

2 McFadden K A, Huang J, Chu X, et al. Pulsed oxidation and biological evolution in the Ediacaran Doushantuo Formation. Proc Natl Acad Sci USA, 2008, 105: 3197-3202

3 Zhou C, Xie G, McFadden K, et al. The diversification and extinction of Doushantuo-Pertatataka acritarchs in South China: Causes and biostratigraphic significance. Geol J, 2007, 42: 229-262

4 Xiao S, Laflamme M. On the eve of animal radiation: Phylogeny, ecology and evolution of the Ediacara biota. Trend Ecol Evol, 2008, 24: $31-40$

5 Zhou C, Brasier M D, Xue Y. Three-dimensional phosphatic preservation of giant acritarchs from the terminal Proterozoic Doushantuo Formation in Guizhou and Hubei provinces, South China. Palaeontology, 2001, 44: 1157-1178

6 Cohen P A, Knoll A H, Kodner R B. Large spinose microfossils in Ediacaran rocks as resting stages of early animals. Proc Natl Acad Sci USA, 2009, 106: 6519-6524

7 Grotzinger J P, Fike D A, Fischer W W. Enigmatic origin of the largest-known carbon isotope excursion in Earth's history. Nat Geosci, 2011, 4: 285-292

8 Jiang G, Shi X, Zhang S, et al. Stratigraphy and paleogeography of the Ediacaran Doushantuo Formation (ca. 635-551 Ma) in South China. Gondwana Res, 2011, 19: 831-849

9 Bristow T F, Kennedy M J, Derkowski A, et al. Mineralogical constraints on the paleoenvironments of the Ediacaran Doushantuo Formation. Proc Natl Acad Sci USA, 2009, 106: 13190-13195

10 Vernhet E, Reijmer J J G. Sedimentary evolution of the Ediacaran Yangtze platform shelf (Hubei and Hunan provinces, Central China). Sediment Geol, 2010, 225: 99-115 
11 McFadden K A, Xiao S, Zhou C, et al. Quantitative evaluation of the biostratigraphic distribution of acanthomorphic acritarchs in the Ediacaran Doushantuo Formation in the Yangtze Gorges area, South China. Precambrian Res, 2009, 173: 170-190

12 Shen B, Xiao S, Zhou C, et al. Yangtziramulus zhangi new genus and species, a carbonate-hosted macrofossil from the Ediacaran Dengying Formation in the Yangtze Gorges area, South China. J Paleontol, 2009, 83: 575-587

13 Xiao S, Shen B, Zhou C, et al. A uniquely preserved Ediacaran fossil with direct evidence for a quilted bodyplan. Proc Natl Acad Sci USA, 2005, 102: 10227-10232

14 Zhou C, Xiao S. Ediacaran $\delta^{13} \mathrm{C}$ chemostratigraphy of South China. Chem Geol, 2007, 237: 89-108

15 Zhu M, Gehling J G, Xiao S, et al. Eight-armed Ediacara fossil preserved in contrasting taphonomic windows from China and Australia. Geology, 2008, 36: 867-870

16 German C R, Elderfield H. Application of the Ce anomaly as a paleoredox indicator: The ground rules. Paleoceanography, 1990, 5: 823833

17 Webb G E, Kamber B S. Rare earth elements in Holocene reefal microbialites: A new shallow seawater proxy. Geochim Cosmochim Acta, 2000, 64: 1557-1565

18 Jiang G, Kaufman A J, Christie-Blick N, et al. Carbon isotope variability across the Ediacaran Yangtze platform in South China: Implications for a large surface-to-deep ocean $\delta^{13} \mathrm{C}$ gradient. Earth Planet Sci Lett, 2007, 261: 303-320

19 Yin C, Liu P, Awramik S M, et al. Acanthomorph biostratigraphic succession of the Ediacaran Doushantuo Formation in the east Yangtze Gorges, South China. Acta Geol Sin, 2011, 85: 283-295

20 Zhu M, Zhang J, Yang A. Integrated Ediacaran (Sinian) chronostratigraphy of South China. Palaeogeogr Palaeoclimatol Palaeoecol, 2007, 254: 7-61

21 Zhao Y Y, Zheng Y F, Chen F. Trace element and strontium isotope constraints on sedimentary environment of Ediacaran carbonates in southern Anhui, South China. Chem Geol, 2009, 265: 345-362

22 German C R, Holliday B P, Elderfield H. Redox cycling of rare earth elements in the suboxic zone of the Black Sea. Geochim Cosmochim Acta, 1991, 55: 3553-3558

23 Komiya T, Hirata T, Kitajima K, et al. Evolution of the composition of seawater through geologic time, and its influence on the evolution of life. Gondwana Res, 2008, 14: 159-174

24 Shields G, Stille P. Diagenetic constraints on the use of cerium anomalies as palaeoseawater redox proxies: An isotopic and REE study of Cambrian phosphorites. Chem Geol, 2001, 175: 29-48

25 Sawaki Y, Ohno T, Tahata M, et al. The Ediacaran radiogenic $\mathrm{Sr}$ isotope excursion in the Doushantuo Formation in the Three Gorges area, South China. Precambrian Res, 2010, 176: 46-64

26 Halverson G P, Dudás F Ö, Maloof A C, et al. Evolution of the ${ }^{87} \mathrm{Sr} /{ }^{86} \mathrm{Sr}$ composition of Neoproterozoic seawater. Palaeogeogr Palaeoclimatol Palaeoecol, 2007, 256: 103-129

27 Elderfield H, Whitfield M, Burton J D, et al. The oceanic chemistry of the rare-earth elements [and discussion]. Philos Trans R Soc Lond Ser A Mathemat Phys Sci, 1988, 325: 105-126

28 Bolhar R, Van Kranendonk M J. A non-marine depositional setting for the northern Fortescue Group, Pilbara Craton, inferred from trace element geochemistry of stromatolitic carbonates. Precambrian Res, 2007, 155: 229-250

29 Sholkovitz E R. Chemical evolution of rare earth elements: Fraction- ation between colloidal and solution phases of filtered river water. Earth Planet Sci Lett, 1992, 114: 77-84

30 Frimmel H E. Trace element distribution in Neoproterozoic carbonates as palaeoenvironmental indicator. Chem Geol, 2009, 258: 338-353

31 Canfield D E, Poulton S W, Knoll A H, et al. Ferruginous conditions dominated later Neoproterozoic deep-water chemistry. Science, 2008, 321: 949-952

32 Li C, Love G D, Lyons T W, et al. A stratified redox model for the Ediacaran ocean. Science, 2010, 328: 80-83

33 Shen Y N, Zhang T G, Hoffman P F. On the coevolution of Ediacaran oceans and animals. Proc Natl Acad Sci USA, 2008, 105: 7376-7381

34 Yuan X, Chen Z, Xiao S, et al. An early Ediacaran assemblage of macroscopic and morphologically differentiated eukaryotes. Nature, 2011, 470: 390-393

35 Zhou C, Jiang S-Y. Palaeoceanographic redox environments for the lower Cambrian Hetang Formation in South China: Evidence from pyrite framboids, redox sensitive trace elements, and sponge biota occurrence. Palaeogeogr Palaeoclimatol Palaeoecol, 2009, 271: 279-286

36 Wright J, Schrader H, Holser W T. Paleoredox variations in ancient oceans recorded by rare earth elements in fossil apatite. Geochim Cosmochim Acta, 1987, 51: 631-644

37 Chen D F, Dong W Q, Qi L, et al. Possible REE constraints on the depositional and diagenetic environment of Doushantuo Formation phosphorites containing the earliest metazoan fauna. Chem Geol, 2003, 201: 103-118

38 Kaufman A J, Jiang G, Christie-Blick N, et al. Stable isotope record of the terminal Neoproterozoic Krol platform in the Lesser Himalayas of northern India. Precambrian Res, 2006, 147: 156-185

39 Le Guerroué E, Allen P A, Cozzi A. Chemostratigraphic and sedimentological framework of the largest negative carbon isotopic excursion in Earth history: The Neoproterozoic Shuram Formation (Nafun Group, Oman). Precambrian Res, 2006, 146: 68-92

40 Walter M R, Veevers J J, Calver C R, et al. Dating the 840-544 Ma Neoproterozoic interval by isotopes of strontium, carbon, and sulfur in seawater, and some interpretative models. Precambrian Res, 2000, 100: $371-433$

41 Grey K. Ediacaran palynology of Australia. Mem Assoc Australian Palaeontol, 2005, 31: 1-439

42 Yin L, Zhu M, Knoll A H, et al. Doushantuo embryos preserved inside diapause egg cysts. Nature, 2007, 446: 661-663

43 Butterfield N J. Plankton ecology and the Proterozoic-Phanerozoic transition. Paleobiology, 1997, 23: 247-262

44 Pokrovskii B G, Melezhik V A, Bujakaite M I. Carbon, oxygen, strontium, and sulfur isotopic compositions in late Precambrian rocks of the Patom Complex, Central Siberia: Communication 1. Results, isotope stratigraphy, and dating problems. Lithol Miner Resour, 2006, 41: 450-474

45 Vorob'eva N G, Sergeev V N, Chumakov N M. New finds of Early Vendian microfossils in the Ura Formation: Revision of the Patom Supergroup age, middle Siberia. Doklady Earth Sci, 2008, 419A: $411-416$

46 Kimura H, Watanabe Y. Oceanic anoxia at the Precambrian-Cambrian boundary. Geology, 2001, 29: 995-998

47 Schröder S, Grotzinger J P. Evidence for anoxia at the EdiacaranCambrian boundary: The record of redox-sensitive trace elements and rare earth elements in Oman. J Geol Soc, 2007, 164: 175-187

Open Access This article is distributed under the terms of the Creative Commons Attribution License which permits any use, distribution, and reproduction in any medium, provided the original author(s) and source are credited. 


\section{Supporting Information}

Figure S1 Petrographic and outcrop photographs of Member III dolostone and limestone at Jiulongwan.

Figure S2 Petrographic and outcrop photographs of Member III dolostone at Tianjiayuanzi.

Figure S3 NASC-normalized REE distribution patterns of carbonate samples from the Jiulongwan (prefix 05HND-) and Tianjiayuanzi (prefix 08TJYZ-) sections.

Figure S4 Correlations of $\mathrm{Zr}$ concentrations vs. Y/Ho ratios, $\mathrm{Zr}$ concentrations vs. $\Sigma \mathrm{REE}$, and $\mathrm{Zr}$ concentrations vs. Ce/Ce* of the studied carbonate samples from Tianjiayuanzi and Jiulongwan sections.

Figure S5 Correlations of $\mathrm{Ce} / \mathrm{Ce} *$ and $\Sigma \mathrm{REE}$ of Jiulongwan carbonate samples treated with $0.5 \mathrm{~N}$ and $1.0 \mathrm{~N}$ acetic acid.

Figure S6 Plots of Ce/Ce* vs. Pr/Pr*.

Table S1 REE data treated with $0.5 \mathrm{~N}$ acetic acid for member III carbonates of the Doushantuo Formation at Jiulongwan (prefix 05HND) and Tianjiayuanzi (prefix 08TJYZ)

The supporting information is available online at csb.scichina.com and www.springerlink.com. The supporting materials are published as submitted, without typesetting or editing. The responsibility for scientific accuracy and content remains entirely with the authors. 